\title{
Pain Management in Patients with Head and Neck Carcinoma
}

\author{
${ }^{1}$ Aditi Ghei, ${ }^{2}$ Sharmila Khot \\ ${ }^{1}$ Specialist Registrar in Anesthesia and Pain Medicine, Department of Anesthesia and Intensive Care Medicine, University \\ Hospital of Wales, Heath Park, Cardiff, CF14 4XN \\ ${ }^{2}$ Consultant and Senior Lecturer in Anesthesia and Pain Medicine, Department of Anesthesia and Intensive Care Medicine \\ University Hospital of Wales, Heath Park, Cardiff, CF14 4XN
}

Correspondence: Sharmila Khot, Consultant and Senior Lecturer in Anesthesia and Pain Medicine, Department of Anesthesia and Intensive Care Medicine, University Hospital of Wales, Heath Park, Cardiff, CF 14 XXN

\begin{abstract}
Pain is one of the feared effects of advancing cancer and can affect a vast majority of head and neck cancer (HNC) patients. HNC's have a devastating impact on patient's lives as both disease and treatment can affect the ability to speak, swallow and breathe due to involvement of the aerodigestive tract. The etiology of pain in this subgroup of patients could be as a result of direct tumor progression, nerve root compression or as a result of the treatment modalities including surgery radiotherapy and chemotherapy. Pain experienced could be nociceptive or neuropathic. A thorough initial and ongoing assessment of the patient is essential for diagnosis and planning the treatment bearing in mind its modification during treatment. Treatment involves a comprehensive approach which is centred around systematic pharmacotherapy, and supplemented by a range of therapeutic interventions and physical and behavioral approaches. In this article we discuss the incidence, etiology, assessment and treatment of this challenging problem using current modalities.
\end{abstract}

Keywords: Cancer pain, head and neck cancer.

\section{INCIDENCE}

Head and neck cancers account for 5 to $10 \%$ of all malignant tumors, ${ }^{1}$ but are a major cause of morbidity and mortality in these patients. After excluding lip and larynx cancers, only $35 \%$ of head and neck malignancies are cured. ${ }^{2}$ Even non fatal, head and neck cancers have a devastating impact on patient's lives as both disease and treatment can affect the ability to speak, swallow and breathe.

Pain is one of the most feared consequences of carcinoma $a^{3,4}$ and is experienced by up to $80 \%$ of patients with head and neck carcinoma (HNC). ${ }^{5,6}$ One of the patients who was dying of cancer stated, "I found that when I didn"t have pain I could forget that I had cancer" "While patients may be able to ignore enlarged nodes, coughing, hoarseness, painless lumps or lesions, tumor related pain often drives them to seek treatment. The incidence of pain as a presenting symptom in patients having tumors of the oral cavity is cited at 20\%. Pain gets further exacerbated during treatments like surgery and chemotherapy and most patients with advanced HNC deal with pain on a daily basis which has a huge impact on their quality of life.

\section{ETIOLOGY OF PAIN IN HNC}

Most patients with advanced HNC have more than one cause of pain. Pain may be due to the cancer itself, effects of the treatments (surgery, radiotherapy or chemotherapy) other debilitating conditions or pain unrelated to the cancer. ${ }^{8}$ Although the physical burden of cancer pain is devastating it is almost always accompanied by emotional, spiritual, financial and social stresses. Therefore a multifaceted approach towards pain management is essential.

Pain is most commonly caused by direct tumor involvement including ulceration, deep infiltration, early metastasis, tumor invasion of bone and distension of pain sensitive structures in soft tissues of the head and neck. ${ }^{3}$ Tumor invasion of bone is a major cause of pain in all cancer patients.

The second most common cause of pain is tumor compression or infiltration of nerve roots trunks or plexuses. Involvement of mucosal or submucosal nerve endings can cause burning, uncomfortable dysesthesias and referred pain like otalgias. ${ }^{3}$ Facial pain along the distribution of the trigeminal nerve particularly the mandibular division is common. ${ }^{9}$ 
Finally pain can occur as a consequence of various treatment modalities such as surgery, radiotherapy or chemotherapy.

\section{TYPES OF HNC PAIN}

Cancer related pain can be classified into two main types, nociceptive and neuropathic.

Nociceptive pain is usually well localized and described as dull, sharp, aching or throbbing. It is caused by acute tissue injury or by noxious mechanical, thermal or chemical stimuli. This leads to sensitization of peripheral nociceptors which are free nerve endings of the A-delta and C-fibers. Tissue injury leads to inflammation which leads to local release of "inflammatory soup" which consists of bradykinin, prostaglandins, substance $\mathrm{P}$, serotonin and other inflammatory mediators and leads to further sensitization of peripheral nociceptors. These pain impulses generated peripherally are transmitted to the dorsal horn of the spinal cord via the A-delta and C-fibers, and then further centrally. Nociceptive pain usually responds to NSAIDS (prostaglandins) $)^{10}$ and opioids (C-fiber related pain).

Neuropathic pain may be described as burning, stabbing, shooting, electric shock like and may be associated with local color and temperature changes.

The International Association of Study of Pain (IASP) defined neuropathic pain as "pain caused by a lesion or dysfunction of the nervous system." 11

In cases of cancer this may be due to nerve compression or invasion of the nerve by the tumor. Regeneration of nerves after damage can lead to neuroma formation and uncontrolled neuronal firing which may be mediated through increased expression of both sodium and voltage-gated calcium channels. Neuropathy generates a local accumulation of sodium channels, with a consequent increase of density. This remodelling appears to be the basis of neuronal hyperexcitability. ${ }^{12}$ Calcium channel expression in the spinal cord leads to increased release of glutamate which is an excitatory neurotransmitter. Hence these channels are the targets for drugs in order to alter expression of neuropathic pain.

Pain can also be classified as acute, intermittent or chronic.

Chronic pain is the most common type of pain in HNC patients and can lead to the associated symptoms of disturbed sleep, decreased appetite, impaired concentration and irritability leading to physical deterioration, emotional and behavioral change. ${ }^{2}$

\section{EVALUATION OF PAIN IN PATIENTS' WITH HNC}

Pain is a subjective phenomenon which requires assessment. A thorough assessment of the patient's pain is essential to establish the cause and type of pain before planning its treatment. Additionally, regular assessment on self reporting of new pain acts as a trigger for changing therapy or introducing interventions as required.

A thorough history and physical examination in the context of the known or suspected primary or secondary site of tumor including an assessment of the patients psychosocial status is required to establish a clinical diagnosis of pain.

History provides valuable information:

- Site: Corresponds to injured area or shows referred pain.

- Onset and pattern of the pain: Classifies pain as acute, intermittent, chronic or incident.

- Description of the pain: Differentiates nociceptive from neuropathic pain.

- Severity: Denotes effectiveness of pain treatments.

- Aggravating or relieving factors: Supports selection and evaluation of analgesic regimen.

- Previous treatments: Inadequate relief from previous treatment may indicate incorrect diagnosis, disease progression or treatment failure.

- Psychological and social assessment: Supports holistic approach to pain management.

Physical examination involves inspection of skin, oral cavity, paranasal sinuses, larynx and thyroid gland.

Questionnaires like brief pain inventory (BPI) and hospital anxiety and depression scale (HADS) are useful tools as they provide an objective analysis of the patients pain and hence a baseline for further treatment. Additionally, the Leeds assessment of neuropathic signs and symptoms (LANSS) questionnaire is useful for identification of neuropathic pain in these patients.

\section{TREATMENT OF HNC PAIN}

The goal of cancer pain treatment is to relieve pain by modifying its source, interrupting transmission or modulating its influence on the brain. Pain in HNC patients is multifactorial and hence requires a comprehensive and multimodal approach. This can be achieved by a combination of treatment modalities (Table 1).

\section{Antineoplastic Therapies}

Decreasing tumor burden by primary antineoplastic therapies remains paramount in the treatment of pain in HNC patients. 
TABLE 1: Pain management options in head and neck cancer

Antineoplastic treatment

- Surgery

- Radiotherapy

- Chemotherapy

Pharmacological management

- NSAIDS

- Opioid's

- Adjuvant analgesics

Interventional pain management

Behavioral pain management techniques

Physical therapies (aromatherapy, TENS, relaxation, hypnosis, acupuncture)

Home based and hospice care

The three main types of treatment for managing head and neck cancer are radiation therapy, surgery and chemotherapy. The primary treatments are radiation therapy or surgery, or both combined; chemotherapy is often used as an additional, or adjuvant, treatment. As discussed previously these antineoplastic therapies almost always lead to pain themselves which will require further management. Surgery especially if it involves the larynx, mouth and tongue can be mutilating and may require the reconstruction with 'flaps' including forearm and lower limb grafts. ${ }^{13}$

\section{Pharmacological Therapy}

Pharmacological therapy is the foundation of cancer pain control. Eighty to ninety percent of cases of cancer pain can be adequately managed through the conceptual analgesic ladder (World Health Organisation, Geneva, 1986) (Fig. 1) which recommends that nonopioid or NSAIDS should be used first and followed, if needed by opioids complemented by analgesic adjuvants. The conceptual analgesic ladder is simple to use, well-validated and effective for cancer pain in general and head and neck cancer in particular. ${ }^{8}$

\section{Non-narcotic Analgesics}

Non-narcotic analgesics are the first line agents for the management of mild to moderate cancer pain and in severe pain potentiate the analgesic effects of narcotic analgesics. ${ }^{3}$ NSAIDS, unless contraindicated, are recommended as the first step on the conceptual pain ladder. These drugs provide analgesia by inhibiting the formation of prostaglandins which are known to sensitize peripheral nociceptors and may play a role in the central nervous system processing of pain signals. ${ }^{14}$ There is evidence to suggest that these medications have a unique role in managing pain from bony metastasis. ${ }^{15}$ Non-narcotic analgesics have a ceiling effect and their longterm use is limited by gastrointestinal and hematological

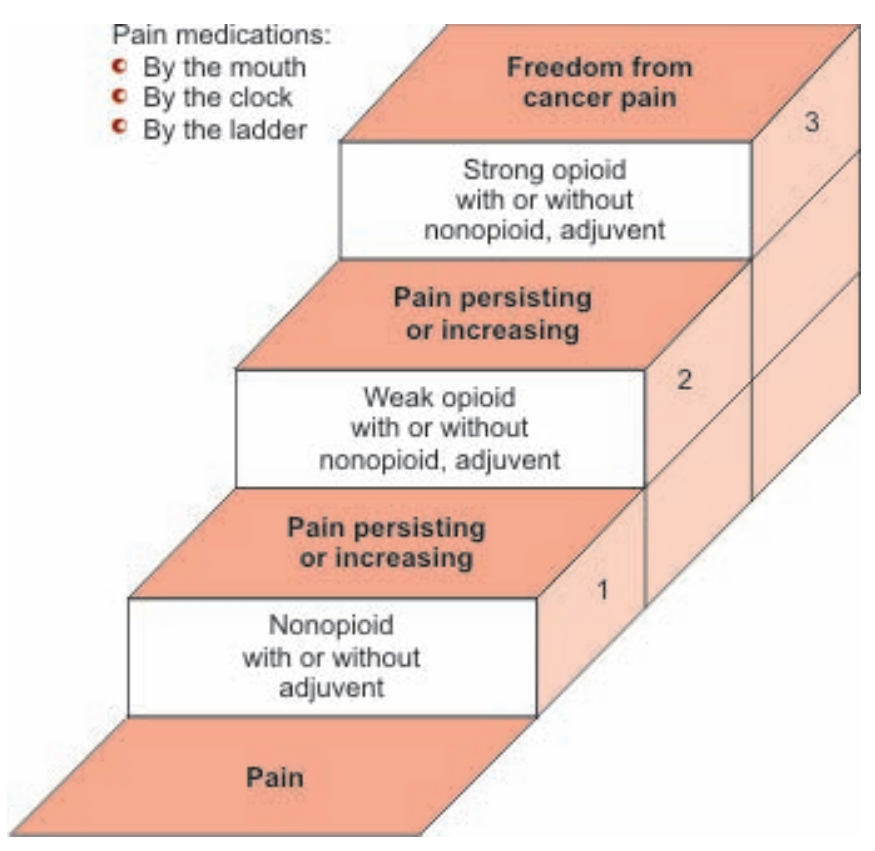

FIGURE 1: WHO pain ladder

side effects. Acetaminophen may be considered a NSAID although it has primarily central effects. Evidence for the relative efficacy of analgesics can be obtained from the Oxford league table of analgesics in acute pain. ${ }^{10}$ The choice and use of NSAIDS should be individualized with the patient receiving maximum doses of one drug before another is tried. If pain control is ineffective or non-narcotic agents are poorly tolerated then the use of narcotic analgesics is indicated.

\section{Narcotic Analgesics}

Opioid analgesics are the cornerstone of pharmacological therapy and occupy the remaining rungs of the conceptual pain ladder. As per the WHO pain ladder, if pain persists after initial treatment with non opioids, or if at presentation is moderate to severe opioids should be introduced. Initially weak opioids such as codeine or tramadol should be prescribed alone or in combination with acetaminophen. If maximum dosages are reached weak opioids are rotated to strong opioids, which include morphine, oxycodone, hydromorphone, fentanyl and methadone. Although meperidine is a strong opioid it is not used for cancer pain due to concerns regarding accumulation of normeperidine and lowering of seizure threshold. ${ }^{16}$

Head and neck cancer patients have unique pain management problems. Involvement of the aerodigestive tract by disease or treatment often renders the oral route unavailable and hence versatility of the routes of 
administration of opioids, coupled with lack of ceiling effects and predictable and manageable side effects makes them the most important analgesics available to the treating physician.

\section{Opioids in Management of Acute Head and Neck Cancer Pain}

Head and neck cancer patients frequently have acute pain which may be the presenting complaint, or related to antineoplastic therapy. In addition acute pain may be a sign of recurrence of cancer.

Guidelines for management of pain in the postoperative period have been published. ${ }^{17}$ The intravenous route is preferred until oral route can be established. A starting dose of morphine is $5-10 \mathrm{mg}$ every 3-4 hours is recommended for adults weighing $>111$ pounds. A patient controlled analgesia device is useful in the postoperative period. ${ }^{18}$

Patients who are managed by radiation or chemotherapy may have changing pain and require narcotics for optimal control of pain.

\section{Opioids in Management of Chronic Cancer Patients}

There are important differences in managing acute pain and chronic cancer pain primarily due to altered pharmacodynamics due to drug tolerance. Chronic cancer patients who have been taking opioids for more than a few weeks are considered opioid tolerant. In the past, there were concerns regarding addiction with the long-term use of opioids in cancer patients. However, studies with patterns of opioid use in cancer patients and other medical illnesses demonstrate that tolerance and physical dependence occur but psychological dependence (addiction) is rare. ${ }^{19,20}$

The principles of use of opioids in cancer pain and as recommended by WHO (1996) are listed in Table 2.

Opioids via oral route: Opioid analgesics should be given at fixed intervals for best results. This provides more continuous pain relief as patients stay within their therapeutic window. Most short acting opioids have a half life of 3 to 4 hours when given orally and a dose should be given round the clock, 4 hourly. ${ }^{21}$ However 'Breakthrough pain', i.e. pain experienced despite round the clock analgesia, may occur despite regular opioid dosing and typically the same opioid is used for regular and breakthrough pain. When initiating an opioid, the breakthrough dose is calculated at
TABLE 2: Principles of opioid use in cancer patients
By the mouth
By the clock
By the ladder
For the individual
Attention to detail

Data from World Health Organization, Cancer Pain Relief (ed 2), 1996.

least half of the routine 4 hourly dose or 10 to $20 \%$ of the total daily dose of opioid. ${ }^{22}$ If multiple doses are required for breakthrough pain, several days in a succession, the round the clock dose should be increased by the amount of opioid given in rescue doses in 24 hours. ${ }^{18}$ If pain stabilizes on a short acting opioid, the long acting equivalent may be substituted. The availability of breakthrough doses does not change.

Other routes of opioid administration: Wherever possible, opioids should be administered by mouth which is the least invasive and least expensive route. Wherever oral route is not available as in advanced HNC, rectal or transdermal route may be used. The mechanism of action of the transdermal opioids available (Fentanyl and Buprenorphine) is that low molecular weight lipophilic molecules contained in a drug reservoir, migrate across the skin into the capillary blood stream because of a concentration gradient.

Skaer et al (2006) suggests an algorithm using the equivalence between $60 \mathrm{mg} /$ day morphine and a $25 \mu \mathrm{g} / \mathrm{hr}$ patch of fentanyl with titration upwards at 24 hours if required. ${ }^{23}$ Pain relief from a fentanyl patch begins 8 to 12 hours after application and oral opiates should be utilized for pain control during the transition period. The patch needs to be changed every 72 hours. The elimination half life is 12 to 22 hours once a patch is removed. Oral transmucosal fentanyl citrate (OTFC) (introduced 1998) or Fentanyl buccal tablets (FBT) (introduced 2006) may be used for management of breakthrough pain. ${ }^{24}$

Transdermal buprenorphine may also be used for management of cancer pain with oral morphine used for breakthrough pain.

Opioid use is limited by their side effects-respiratory depression, sedation, pruritis, nausea and vomiting, urinary retention and constipation. The occurrence of these side effects should be anticipated and treated. Most of these side effects except sedation and constipation decrease with time. A stool softener and laxative should always be 
prescribed with initiation of opioid therapy. ${ }^{14}$ At any stage a non opioid may be prescribed to potentiate the effect of the opioid and hence decrease the dose to avoid side effects.

\section{Adjuvant Analgesics}

A number of other classes of drugs can be used to enhance the effect of narcotic and non-narcotic medication. These include antidepressants, anticonvulsants, local anesthetic plasters and steroids as the most common ones.

Drugs used for neuropathic pain: The neuropathic component of cancer pain is frequently underdiagnosed or inadequately treated or both. Patients may describe the pain as burning, shooting stabbing, radiating or aching in the dermatomal distribution of the damaged neural structure. Although used widely in cancer pain opioids may have limited efficacy in the management of neuropathic pain. ${ }^{25-27}$

Finnerup et al in 2005 formulated a treatment algorithm for neuropathic pain on the basis of a review of 105 highquality, randomized, placebo-controlled clinical trials. They concluded TCAs had the lowest numbers needed to treat (NNT) followed by opioids and antiepileptic drugs such as gabapentin and pregabalin. ${ }^{28}$

Amitryptyline (Tricyclic antidepressant) can produce direct analgesic effect through blocking serotonin and noradrenalin reuptake at CNS synapses. It can be started at a dose of 10 to $25 \mathrm{mg}$ at bedtime and increased to 50 to $150 \mathrm{mg}$ at bedtime. Its use is limited by sedative and anticholinergic side effects (dry mouth, urinary retention, delirium).

Gabapentin is an anticonvulsant drug licensed for use in neuropathic pain. It acts centrally at dorsal horn neurons by binding to calcium channels. Gabapentin is usually started a low dose and then titrated upwards, as necessary to achieve pain relief and as tolerated be the patient. It is usually prescribed to be taken three times a day. Gabapentin is excreted unchanged by the kidneys and hence dosage adjustments are required in patients with renal failure. Data from a recent (2007) randomized trial on cancer patients with neuropathic pain, suggests that gabapentin added to an opioid provides better relief of neuropathic pain in cancer patients than opioid monotherapy. ${ }^{29}$

Pregabalin is an anticonvulsant similar in structure to gabapentin which was designed to have greater affinity for the same calcium channels blocked by gabapentin. The linear pharmacokinetics of pregabalin allow for twice daily dosing and rapid titration as compared to gabapentin. ${ }^{30}$
Corticosteroids possess analgesic properties for several cancer pain syndromes including both neuropathic and bone pain. ${ }^{31}$ The risk of adverse effects increases with dose and duration of therapy.

\section{INTERVENTIONAL PAIN MANAGEMENT}

Among the head and neck cancer patients, 10 to $20 \%$ do not obtain satisfactory pain relief or get unacceptable side effects from systemic pharmacological therapy. It is in this group of patients that invasive techniques such as nerve blocks, neurodestructive procedures and intraspinal/ intrathecal drug infusions may be indicated. ${ }^{14}$

Cranial and peripheral nerves which may be commonly blocked either centrally or peripherally are trigeminal, glossopharyngeal, occipital, vagus and branches of the cervical plexus. ${ }^{14}$ However, sensory innervations may overlap due to anatomic distortion due to cancer, surgery or radiotherapy. Occipital nerve block is the treatment for the symptomatic management of occipital headaches which may be the manifestation of base of skull invasion by cancer. Myofacial pain may be present in the area of trapezius or the sternocleidomastoid which may respond to trigger point injections. Pain in the $\mathrm{C} 2, \mathrm{C} 3$ dermatomal distribution following radical neck dissection may be alleviated by superficial cervical plexus block.

A 3-stage process involving diagnostic, prognostic, and therapeutic blocks is recommended to ensure appropriate patient selection and therapeutic efficacy. ${ }^{32}$ Diagnostic nerve blocks identify the anatomic source of pain and are best performed using relatively short-acting local anesthetics such as lidocaine $1 \%$ or $2 \%$. Prognostic nerve blocks give patients the opportunity to experience the numbness and other side effects associated with regional blocks in the head and neck.

Injection of a long-acting local anesthetic, such as bupivacaine, enables the patient to feel the sensation of "numbness" that will be associated with the subsequent neurolysis. Local anesthetic blocks can be useful in prognosticating the results of the intended neurolytic block and subsequently, alcohol or phenol may be used for neurolysis. $^{32}$

Chemical or radiofrequency neurolysis has been used for lesions involving trigeminal ganglion and its branches, glossopharyngeal nerve and sphenopalatine ganglion. Varghese et al described a technique of nasal endoscopically guided neurolytic sphenopalatine ganglion block with $6 \%$ phenol after a prognostic block with local anesthetic solution. 
They performed the block in 22 patients with advanced head and neck malignancy and reported good pain relief in 17 patients and inadequate relief in one patient only. ${ }^{33}$ Therefore in well-selected patients with intractable pain neurosurgical destructive procedures such as dorsal rhizotomy or trigeminal tractotomy may have a role. However, these procedures are invasive and have associated morbidity and mortality. The pain relief obtained is often temporary and linked with side effects like sensory and motor dysfunction. Neurosurgical procedures may be technically difficult and pain relief may not occur at all or pain may return after a variable period of time. Following surgery there may be also be nerve and tract regeneration and somatotopographic reorganization including opening of alternate pathways. ${ }^{34}$ Therefore these procedures are not commonly performed currently. ${ }^{11}$

Intrathecal morphine may be used by infusion via implantable delivery systems after a diagnostic trial, to determine pain relief in cases with intractable pain. Sites caudal to the cervical region may cause analgesia in the cervical or cranial regions. ${ }^{14}$ Applegren et al (1996) reported the use of intracisternal and high cervical intrathecal bupivacaine infusion in refractory head and neck pain with very few complications. ${ }^{35}$ However, these treatments are invasive and more experience is needed before such treatments are commonly used in head and neck cancer patients.

\section{Alternative Therapy}

\section{Behavioural Management Techniques}

The aim of behavioral management approaches is to help the patient regain a sense of control which has been undermined by disease and debility.

Behavioral approaches including cognitive behavioral training, hypnosis, reiki biofeedback and relaxation therapies have been used. These techniques serve as a calming diversion of attention breaking the pain-anxiety-tension cycle.

\section{Physical Modalities}

Physical modalities such as TENS and acupuncture may be used in conjunction with other management approaches. A recent randomized control trial (2008) by P fister et al on 70 patients postradical neck dissection for $\mathrm{HNC}$, showed acupuncture to provide pain relief in 39\% patients compared to $7 \%$ standard care group. ${ }^{36}$

\section{CONCLUSION}

Pain is the one of the most feared complications of advancing cancer. In addition the effect is probably magnified in the head and neck cancer patient due to the additional burden of pain on functionality and cosmesis. Assessment of the type and cause of pain in these patients helps clinicians to define proper therapy. The analgesic ladder provides an effective framework which can be modified to suit the needs of particular patients. An understanding of pain pathways and mechanisms allows for addition of adjuvant drugs, interventional treatments and physical and psychological treatments for overall management and compassionate treatment of this subgroup of patients. In conclusion, "Cancer pain should be treated as aggressively as the cancer itself."

\section{REFERENCES}

1. Keefe FJ, Manuel G, Brantley A. Pain and the head and neck cancer patient: Changes over treatment. Head Neck Surg 1986;8:169-76.

2. Raj PP, Phero JC. Pain control in cancer of the head and neck in Thawley SE, Panje WR (Eds): Comprehensive management of Head and Neck Tumors. Philadelphia. PA Saunders. 1987;1:4268.

3. Foley KM. The treatment of cancer pain. N Engl J Med 1985;313:84-95.

4. Olsen KD, Creagan ET. Pain management in advanced carcinoma of the head and neck. Am J Otolaryngol 1991;12:154-69.

5. Lyons MF, Redmond J, Covelli H. Multiple primary neoplasia of the head and neck and lung: The changing histopathology Cancer1986;57:2193-97.

6. Foley KM, Intrinsic CE. Analgesic drug therapy in cancer pain: Principles and practice. Med Clin North Am 1987;71:207-32.

7. McGivney WT, Crooks GM. The care of patients with severe chronic pain in terminal illness. JAMA 1984;251:1182-88.

8. Grond S, Zech D, Lynch J, Diefenbach C, Schug SA, Lehmann KA. Validation of World Health Organisation Guidelines for pain relief in head and neck cancer. A prospective study. Ann Otol Rhinol Laryngol 1993;102:342-48.

9. Shapshay SM, Scott RM, McCann CF, Stoelting I. Pain control in advanced and recurrent head and neck cancer. Otolaryngol Clin North Am 1980;13:551-60.

10. Oxford league table of analgesics in acute pain. Bandolier 2007 [Accessed:26/11/2009] Available from http:// www.medicine.ox.ac.uk/bandolier/booth/painpag/Acutrev/ Analgesics/Leagtab.html.

11. "Part III: Pain Terms, A Current List with Definitions and Notes on Usage" Classification of Chronic Pain, Second Edition, IASP Task Force on Taxonomy, edited by H. Merskey and N. Bogduk, IASP Press, Seattle, (c) 1994;209-14.

12. Aurilio C, Pota C, Pace MC, Passavanti MB, Barbarisi M. Ionic channels and neuropathic pain: Physiopathology and applications. $\mathrm{J}$ of cellular physio 2008;215(1):8-15. 
13. Head and neck cancer. In: Lecture notes: Diseases of Ear Nose Throat. Bull P, Clarke R editors (10th ed). Wiley-Blackwell 2007;142-46.

14. Dennis J, Patin MD. Pain control in head and neck cancer. Current opinion in otolaryngology and head and neck surgery 1998;86:8589.

15. Brodie GN. Indomethacin and bone pain. Lancet 1974;2:1160.

16. Inturrisi CE. Clinical pharmacology of opioids for pain. Clin J of Pain 2002;18(suppl):S3-13.

17. Carr DB, Jaycox A. Acute pain management in adults: Operative procedures. Agency for health care policy and research. US department of health and human services 1992.

18. Thompson AR. Opioids and their proper use as analgesics in the management of head and neck cancer patients. Am J of Otolaryngol 2000;21(4):244-54.

19. Angell M. The quality of mercy. N Eng J Med 1982;306:98-99.

20. Porter J, Hick H. Addiction rare in patients treated with narcotics. N Eng J Med 1980;302:123.

21. MacDonald N. Palliative Medicine: A Case Based Manual (2nd ed). New York: Oxford University Press; 1998

22. Bruera E, Kim HN. Cancer Pain. JAMA 2003;290:2476-79.

23. Skaer TL. Transdermal opioids for cancer pain. Health qual life outcomes 2006;4:24.

24. Darwish M, Messina J. Clinical pharmacology of fentanyl buccal tablet for the treatment of breakthrough pain. Expert rev clin pharmacol 2008;1(1):39-47.

25. Dellemijn P. Are opioids effective in relieving neuropathic pain? Pain 1999;80:453-62.

26. Katz N, Benoit C. Opioids for neuropathic pain. Curr Pain Headache Rep 2005;9:153-60.
27. Arner S, Mayerson BA. Lack of analgesic effect of opioids on neuropathic and idiopathic forms of pain. Pain 1988;33:11-23.

28. Finnerup NB, Otto M, Jenson TS, et al. Algorithm for neuropathic pain treatment: An evidence based proposal. Pain 2005;118:289-305.

29. Keskinbora K, Pelek AF, Aydinli I. Gabapentin plus opioid for managing neuropathic cancer pain. J Pain Symptom Manage 2007;34(2):183-89.

30. Ben-Menachem E. Pregabalin pharmacology and its relevance to clinical practice. Epilepsia 2004;45(6):13-18.

31. Portenoy RK. Adjuvant analgesics in pain management. In: Doyle D, Hanks GWC, MacDonald N (Eds). Oxford Textbook of Palliative Medicine (2nd ed). New York, NY. Oxford University Press 1998.

32. Sist T. Head and neck nerve blocks for cancer pain management. Tech Reg Anesth Pain Manage 1997;1:3-10.

33. Varghese BT, Koshy RC. Endoscopic transnasal neurolytic sphenopalatine ganglion block for head and neck cancer pain. J Laryngol and Otol 2001;115(5):385-87.

34. Tasker RR. The recurrence of pain after neurosurgical procedures. Qual of life research 1994;3(1):S43-49.

35. Appelgren L, Janson M, Nitescu P, Curelaru L. Continuous intracisternal and high cervical intrathecal bupivacaine analgesia in refractory head and neck pain. Anaesthesiology 1996;84:25672.

36. Press release. Acupuncture reduces pain and dysfunction in head and neck cancer patients after neck dissection. 31Mar 2008. (Accessed 19 Nov 2009). Available from: http://www.mskcc.org/ mskcc/html/85411.cfm. 